\title{
Rolling-Sliding Contact Fatigue of Surfaces with Sinusoidal Roughness
}

\author{
Wei Pu' ${ }^{1}$, Dong Zhu ${ }^{1 *}$, Jiaxu Wang ${ }^{1,2}$, and Q. Jane Wang ${ }^{3,2}$ \\ 1. School of Aeronautics and Astronautics \\ Sichuan University, Chengdu 610065, China \\ 2. State Key Laboratory of Mechanical Transmission, \\ Chongqing University, Chongqing 400044, China \\ 3. Center for Surface Engineering and Tribology \\ Northwestern University, Evanston, IL 60208, USA \\ * Corresponding author, DongZhu@Mail.com
}

\begin{abstract}
Surfaces of mechanical components under combined rolling and sliding motions may be subjected to accelerated contact fatigue failure due to increased number of microscopic stress cycles and pressure peak heights caused by rough-surface asperity contacts. Available rolling contact fatigue (RCF) theories were developed mainly for rolling element bearings, for which the effect of sliding is usually insignificant. In various types of gears, however, considerable sliding exist in the critical tooth contact area below the pitch line, where excessive wear and severe pitting failures originate. Ignorance of sliding is most likely the reason why the conventional RCF models often overestimate gear fatigue life. This paper studies the effect of sliding motion on the contact fatigue life of surfaces with sinusoidal roughness that mimicks the topography from certain manufacturing processes. A set of simple equations for stress cycle counting is derived. Mixed elastohydrodynamic lubrication simulations are executed with the considerations of normal loading and frictional shear. Relative fatigue life evaluations based on a subsurface stress analysis is conducted, taking into account the two sliding-induced mechanisms, which are the greatly increased number of stress cycles and the pressure peak heights due to surface interactions. Obtained results indicate that sliding leads to a significant reduction of contact fatigue life, and rough surface asperity contacts result in accelerated pitting failure that needs to be considered in life predictions for various mechanical components.
\end{abstract}

\section{Keywords:}

Rolling-Sliding Contact, Elastohydrodynamic Lubrication (EHL), Mixed EHL, Contact Fatigue, Contact Fatigue Life Prediction 


\section{INTRODUCTION}

Power transmission is often accomplished through contact of component surfaces under combined rolling and sliding motions. Pure rolling contact, often found in rolling element bearings, can be considered as a special case of rolling-sliding contacts. It is well known that surface pitting due to contact fatigue is a major failure mode for many mechanical components subjected to counterformal contacts, such as various types of gears, rolling element bearings, cam and follower systems, continuously variable speed transmissions, and some metal-forming tools. Available rolling contact fatigue (RCF) theories (see [1-5], for example) have been developed mainly for rolling element bearings, where sliding motion is usually insignificant. In various types of gears, however, considerable sliding can be found in critical tooth contact areas below the pitch line, where excessive wear and severe pitting failures originate. Testing results obtained from two-disc experiments have indicated that, under otherwise the same conditions, the reduction of relative sliding from $25 \%$ to $10 \%$, and then down to $0 \%$ may result in a great increase in contact fatigue life by two orders of magnitude, as reported by Bujold et al. [6]. Most recently studies presented by Govindarajan et al. [7], Oksanen et al. [8], Ramalho et al. [9], Lee et al [10], and Seo et al [11], all indicate that sliding appears to have a significant influence on contact fatigue behaviors. Exclusion of the sliding effect is most likely a major reason why the conventional RCF models often overestimate gear pitting life.

It is well known that engineering surfaces are not ideally smooth, and different surface topographic features may lead to different responses to rolling and sliding. In a pure-rolling contact, the number of stress cycles experienced by a certain piece of material on a surface is basically the same as that of the rolling cycles. With the presence of sliding, however, the number of stress cycles may be significantly higher because many asperities of one surface can pass over a given point in the mating surface during each rolling cycle. Apparently, the number of stress cycles should be, in general, a function of slide-to-roll ratio, $S$, defined as $S=2\left(U_{2}-\right.$ $\left.U_{1}\right) /\left(U_{1}+U_{2}\right)$, where $U_{1}$ and $U_{2}$ are the surface velocities of the two rollers, and the properties of rough surface topography.

The fundamental theories for predicting the limiting number of cycles to fatigue under a certain probability were developed by Weibull [1, 2], Bakharev [3], Lundberg and Palmgren [4] and others. More recent phenomenological fatigue models, such as those by Ioannides and Harris 
[5], and Zaretsky [12], et al., have been used to predict the lifetime of rolling elements with an improved accuracy. Furthermore, extensive lifetime data for well-characterized systems are available in the literature. The effects of sliding have received some attention [13-16] through analyzing surface shear and stress histories under the influence of sliding. In addition, the damage-cumulative approach by Ai [17] adopted a variable to modify the probability that the material at a location experiences a certain stress level.

It should be mentioned that the detailed pressure distribution should be obtained from a mixed elastohydrodynamic lubrication (EHL) analysis in order to obtain accurate pressure distributions subjected to rolling-sliding, asperity contact, and interfacial frictional shear, before any one of the above-mentioned life models is used. This is because that, in engineering practice, surface roughness is often of the same order of magnitude as, or even greater than, the average EHL film thickness. Therefore, most mechanical components operate in the mixed EHL regime, in which localized pressure peak heights due to surface asperities may be much higher than the Hertzian pressure, causing significantly increased subsurface stresses and number of stress cycles. Great efforts have been made in order to develop mixed EHL models for rough surface lubrication. Representative studies include those by Xu and Sadeghi [18], Zhu and Ai [19], Jiang et al. [20], Holmes et al. [21, 22], Zhu et al. [23], and others. The first unified mixed EHL model for point contact problems with 3-dimensional (3D) machined roughness was presented by Zhu and Hu, 1999 [24], Hu and Zhu, 2000 [25], and Zhu [26] et al., which has been demonstrated to be capable of simulating the entire transition from the full-film and mixed lubrication all the way down to the boundary lubrication and dry contact. This mixed EHL model and the subsurfacestress-based fatigue-life model by Zaretsky [12] have resulted in effective approaches for pitting life predictions by Epstein et al. [27], Zhu et al. [28], Greco et al [29], and others. However, no sliding effect was considered in these studies.

The present study aims to investigate the critical effect of relative sliding motion on contact fatigue life, due to the increased number of stress cycles and the high asperity contact pressure, for concentrated contacts in mixed lubrication without considering wear. This paper reports the development of an equation set for stress cycle counting, the execution of mixed EHL simulations, and the comparative analyses of near-surface fatigue under $50 \%$ possibility. The variations of the mixed EHL pressure distribution and asperity stress cycle as a result of the $S$ 
increase are analyzed. The effects of sliding on pressure distribution, friction, subsurface stress cycles, and fatigue life of surfaces with sinusoid-like roughness are numerically investigated.

\section{ASPERITY STRESS CYCLE COUNTING}

The contact of two rollers (cylindrical, spherical, or elliptical in shape) shown in Fig.1 is a generic geometric representation of two surfaces in a non-conformal sliding-rolling interface. The severity of sliding is measured by the slide-to-roll ratio, $S=\left(U_{2}-U_{1}\right) / U$, where $U_{1}$ and $U_{2}$ are the surface velocities of the two rollers, respectively, and the rolling speed $U=\left(U_{1}+U_{2}\right) / 2$. It may be assumed that $U_{2} \geq U_{1}$ without loss of generality. The pure-rolling condition is referred to $S=0$, while $S=2$ defines a simple sliding case in which one surface is stationary and the other moves, as in the cases of the pin-disk contact in a pin-on-disk tribometer, the apex seal contact in a rotary engine, most sliding bearing interfaces, some electrical brush sets and electrical switches, etc.

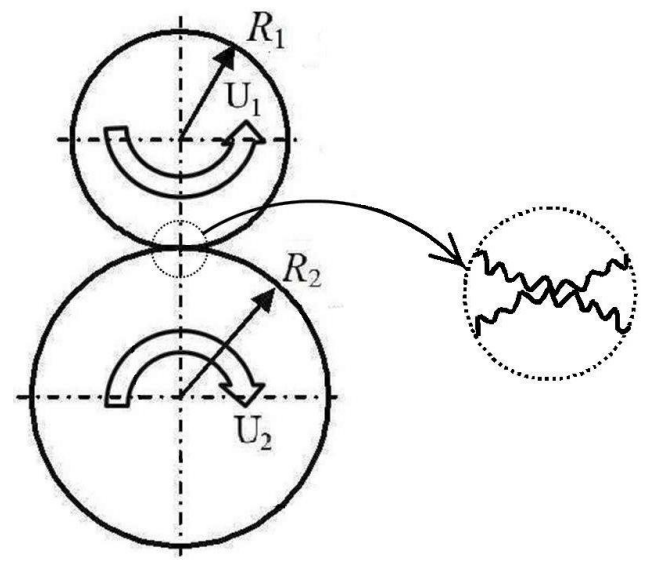

\section{Fig. 1. Contact of Equivalent Cylindrical (or Spherical, or Elliptical) Rollers.}

The topography of an engineering surface often exhibits both periodic and random characteristics due to the machining processes commonly used. Typically the waviness may be treated as periodic and the small-scale roughness may be considered random. A ground surface, chosen from a gear tooth flank, is shown in Fig. 2 as a typical example. The 3D surface topography in the upper graph was scanned by using an optical profilometer, and its topographic profile in the direction of motion and the waviness profile obtained from a low-pass filter are given in the lower graph. It is reasonable to employ sinusoidal waves to approximate such 
machined rough surfaces in mixed EHL simulations and fatigue life predictions in the present work.

Because asperities experience loading and unloading in contact and the mixed lubrication, with and without contact, the stress peak should be the same as the stress range if the residual stress due to plastic deformation is neglected. Furthermore, for transversal roughness, the cycles of the near-surface stress variation should be concurrent with those of the pressure variation. An asperity-passing model, therefore, can be developed to count the actual number of stress cycles. Figure 3 shows the interaction of two rough surfaces, where Surface 2 is supposed to move faster. The number of stress cycles that a certain asperity experiences is related to the effective peak density of the asperities of its mating surface in the interaction range. Note that $f_{1}$ and $f_{2}$ are defined as the asperity peak densities of Surfaces 1 and 2 within the interaction zone, respectively.
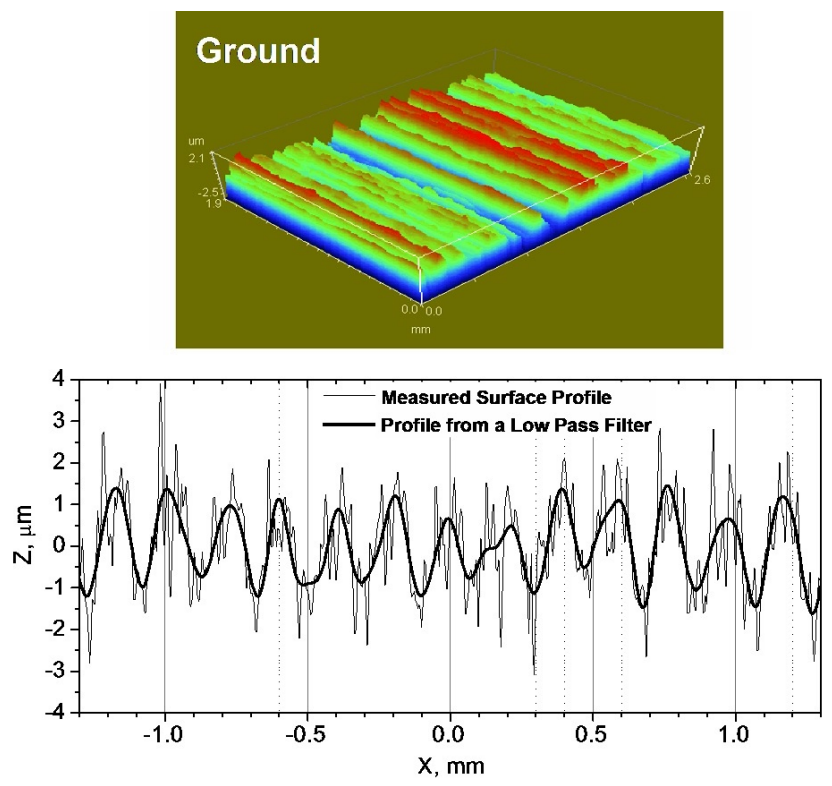

Fig. 2. A Sample Ground Surface.

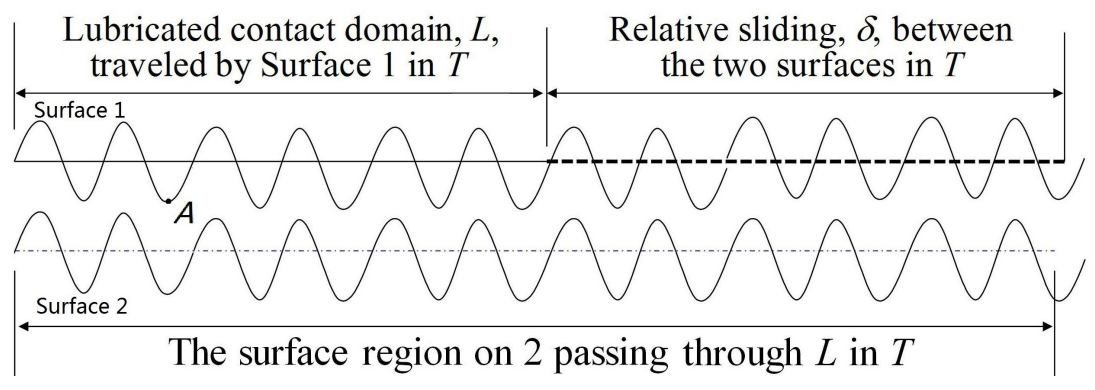

Fig. 3. Effect of Relative Sliding on Asperity Cycle Counting. 
The time required for point $A$ on Surface 1 to pass through the surface interaction zone, $L$, may be expressed as

$$
T=\frac{L}{U_{1}}=\frac{L}{U(1-0.5 S)}
$$

The sliding distance is then given by

$$
\delta=T\left(U_{2}-U_{1}\right)=\frac{L S}{1-0.5 S}
$$

The number of asperity contacts during one loading cycle experienced by point $A$ can be estimated as

$$
n \approx 1+\delta \times f_{2}=1+\frac{L f_{2} S}{1-0.5 S}
$$

Equation (3) can be extended to include cases in which $U_{1}>U_{2}$ as follows:

$$
n \approx 1+\frac{L f_{2}|S|}{1-0.5 S}
$$

where, according to the above definition, $S$ is negative when $U_{1}>U_{2}$. Note that the number of asperity contacts per loading cycle does not depend on individual surface speeds, but only on the slide-to-roll ratio $S$. The factor $L f_{2}$ appearing in Eq. (4) is the number of asperities on Surface 2 that fits within the interaction zone. If, for example, $L f_{2}=8$ and $S=0.4$, then the number of asperity contacts per loading cycle passing through point $A$ on Surface 1 is $n=5$.

The rate of asperity contacts experienced by point $A$ within the interaction zone can be calculated as

$$
\dot{n}=n / T=\frac{U}{L}\left(1-0.5 S+L f_{2} S\right) \text { inside } L
$$

Under pure rolling conditions $\left(S=0, U_{1}=U_{2}\right)$, there is exactly one asperity contact per loading cycle, as is evident from Eq. (4). In simple sliding ( $\left.S=2, U_{1}=0\right)$, the asperities inside the contact region, $L$, of the stationary Surface 1 experience the interaction with all the asperities of Surface 2 at the rate of $U_{2} f_{2}$. 


\section{MIXED EHL SIMULATION AND FATIGUE LIFE PREDICTION}

\subsection{The mixed EHL model}

A mixed EHL model is needed for the prediction of asperity-level pressure distribution and asperity penetration through the lubricant film. Zhu and Hu's unified EHL model [24-26] describing the mixed lubrication of rough surfaces is applied in the present study. The model description has been published in a number of previous papers, but for clarity, its basic formulation will be briefly given below. The pressure within the entire solution domain is governed by the Reynolds equation expressed as:

$$
\frac{\partial}{\partial x}\left(\frac{\rho h^{3}}{12 \eta^{*}} \frac{\partial p}{\partial x}\right)+\frac{\partial}{\partial y}\left(\frac{\rho h^{3}}{12 \eta^{*}} \frac{\partial p}{\partial y}\right)=\frac{u_{1}+u_{2}}{2} \frac{\partial(\rho h)}{\partial x}+\frac{\partial(\rho h)}{\partial t}
$$

where the $\mathrm{x}$-axis is chosen to coincide with the motion direction. The instantaneous lubricant film thickness (or the gap between the two rough surfaces), $h$, is calculated by a geometric equation written as:

$$
h=h_{0}(t)+\frac{x^{2}}{2 R_{x}}+\frac{y^{2}}{2 R_{y}}+v(x, y, t)+\delta_{1}(x, y, t)+\delta_{2}(x, y, t)
$$

in which $R_{x}$ and $R_{y}$ are the local radii of curvature in the x- and y-directions, respectively, $\delta_{1}$ and $\delta_{2}$ denote the roughness height variations of Surfaces 1 and 2 , and $v$ the surface deformation that can be calculated by the Boussinesq integral as follows:

$$
v(x, y, t)=\frac{2}{\pi E^{\prime}} \iint_{\Omega} \frac{p(\xi, \varsigma)}{\sqrt{(x-\xi)^{2}+(y-\varsigma)^{2}}} d \xi d \varsigma
$$

An effective viscosity $\eta^{*}$ has been introduced in Eq. (6) to describe the non-Newtonian lubricant properties. Assuming the variation of viscosity across the lubricant film can be ignored, one can calculate the effective viscosity as follows considering possible shear thinning effect [30]:

$$
\frac{1}{\eta^{*}}=\frac{1}{\eta} \frac{\tau_{0}}{\tau_{1}} \sinh \left(\frac{\tau_{1}}{\tau_{0}}\right)
$$

where $\tau_{o}$ is a reference shear stress given here as $18.0 \mathrm{MPa}$ for a typical mineral oil, $\tau_{1}$ denotes the shear stress acting on Surface 1 , and $\eta$ is the low shear viscosity that is assumed to be a function of pressure, commonly following the exponential relationship: 


$$
\eta=\eta_{0} e^{\alpha p}
$$

The variation of the lubricant density is also dependent on pressure, usually evaluated by:

$$
\rho=\rho_{0}\left(1+\frac{0.6 \times 10^{-9} p}{1+1.7 \times 10^{-9} p}\right)
$$

The applied load is balanced by the integral of the pressure over the entire solution domain:

$$
w(t)=\iint_{\Omega} p(x, y, t) d x d y
$$

The limiting shear stress in the lubricant film should be a function of temperature, and the latter is related to interfacial sliding friction. Consequently, the mutual effects between friction and flash temperature should be taken into account with a non-Newtonian rheological model. It should be pointed out that the effect of rheological models on film thickness and pressure is limited. For friction calculation, the shear stress in the lubricant film can be estimated in the hydrodynamic areas by using the visco-elastic non-Newtonian fluid model developed based on the experimental results by Bair and Winer [31], expressed as:

$$
\dot{\gamma}=\frac{\dot{\tau}}{G_{\infty}}-\frac{\tau_{L}}{\eta} \ln \left(1-\frac{\tau}{\tau_{L}}\right)
$$

in which the limiting shear stress, $\tau_{L}$, and the limiting shear elastic modulus, $G_{\infty}$, are functions of pressure and temperature, which can be estimated empirically. Readers are referred to [30, 32] for details.

It is assumed that the coefficient of friction in the contact area is a constant that can be estimated experimentally. The total friction can then be obtained through the integration of shear stress over the entire domain, including both the hydrodynamic areas and the contact areas as detailed in [32] for evaluating friction in mixed EHL. The calculation of flash temperature is based on the theory of a moving heat source over a semi-infinite solid formulated for mixed EHL [32],

$$
T_{1}(\zeta)=T_{b 1}+\left(\frac{1}{\pi \rho_{1} C_{1} u_{1} k_{1}}\right)^{0.5} \int_{-x}^{\zeta}\left\{\frac{k_{f}}{h}\left[T_{2}(\xi)-T_{1}(\xi)\right]+\frac{q(\xi)}{2}\right\} \frac{d \xi}{(\zeta-\xi)^{0.5}}
$$




$$
T_{2}(\zeta)=T_{b 2}+\left(\frac{1}{\pi \rho_{2} C_{2} u_{2} k_{2}}\right)^{0.5} \int_{-x}^{\zeta}\left\{\frac{k_{f}}{h}\left[T_{1}(\xi)-T_{2}(\xi)\right]+\frac{q(\xi)}{2}\right\} \frac{d \xi}{(\zeta-\xi)^{0.5}}
$$

where $q$ is the heat flux generated by either the lubricant shear in the hydrodynamic areas, or asperity friction in the asperity contact areas.

\subsection{The fatigue life model}

In the present study the RCF life, adopted from Zaretsky [12], is expressed in terms of the number of stress cycles to failure, $M$, corresponding to a given probability of survival (reliability), $P_{S}$ :

$$
\ln \frac{1}{P_{s}} \sim M^{e} \iiint_{V} \sigma_{\text {eff }}^{e c} d V
$$

where $e$ is the Weibull slope, $V$ the stress-affected material volume, $c$ the stress exponent, $\sigma_{\text {eff }}$ the effective stress experienced by the elementary material volume, $d V$. The octahedral stress, which differs from the Mises stress by a factor of $3 / \sqrt{2}$, is used as the effective stress:

$$
\sigma_{\text {eff }}=\frac{1}{3}\left[\left(\sigma_{x}-\sigma_{y}\right)^{2}+\left(\sigma_{y}-\sigma_{z}\right)^{2}+\left(\sigma_{z}-\sigma_{x}\right)^{2}+6\left(\tau_{x y}^{2}+\tau_{y z}^{2}+\tau_{z x}^{2}\right)\right]^{1 / 2}
$$

Detailed discussion on this fatigue model for RCF of surfaces subjected to mixed lubrication can be found in the papers by. Epstein et al. [27] and Zhu et al [28].

In view of the asperity stress cycle adjustment described in the previous section, the number of stress cycles, $M$, in the above fatigue life equation, (16), should not be considered as the number of component loading cycles, or revolutions, as is conventionally assumed in fatigue life models, but rather as the approximate number of actual asperity loading-unloading cycles experienced by a certain stressed volume. In the case of transverse rough surfaces, $M=N \times n$, where $N$ is the number of component loading cycles and $n$ is the number of asperity contacts per loading cycle given by Eq. (4). Here, the length of the interaction zone, $L$, can be equated with the Hertzian contact length, $2 a$. Consequently, $1 / n$ is the life reduction factor introduced by asperity cycle counting, which is a function of $S$ and the asperity density of the mating surface.

Interior stress components are calculated based on the obtained mixed EHL pressure distribution and friction by using the stress equations found in [33], as described in detail in [3435] and some other publications. The stresses away from the contact zone decay rapidly so they 
are ignored. Thus, calculated octahedral stresses can also be regarded as the effective stress range during a contact cycle.

An absolute fatigue life calculation is not meaningful unless all of the material constants in the fatigue life equation, (16), can be determined by independent experiments. However, a relative fatigue life comparison can be readily achieved for contacting surfaces of the same material and the same global geometry for understanding the trend of the influence of sliding on contact fatigue life.

\section{RESULTS AND DISCUSSIONS}

The above mixed EHL model has been validated a number of times by comparing its results with both the experimental data and the previous numerical solutions published by others. A detailed description of the available model validation cases is lengthy and beyond the scope of the present paper; however, two groups of validation cases are shown here for clarity. Two sample cases are selected from [36] as the reference for the first validation group, one with a transversal sinusoidal roughness and the other with longitudinal waves. The main input parameters for this circular contact problem are listed in Table 1 (the symbol definitions are given in the nomenclature). The obtained results are summarized in Fig. 4, showing that the film

thickness and pressure profiles for $x=0$ and $y=0$ from the present model well agree with those from [36].

The experimental results published by Kaneta et al. [37] are chosen as the second-group model validation cases for the situation with significant roughness under combined rollingsliding motions. The main input parameters are given in Table 2, and the rough surface texture used has been described in detail in [37-38]. Figure 5 presents that the simulation results for the film thickness profiles are in a good agreement with those from the optical interferometry presented in [37]. Note that this set of experimental results has been compared once before with the numerical simulations by Liu et al. [38], and satisfactory agreement was also achieved. The mixed EHL model used in [38] was nearly the same as the present one except the difference in the rheological models. 
Table 1. Input Parameters for the Cases from [36]

\begin{tabular}{|c|c|c|c|}
\hline$R_{x}$ & $12.7 \mathrm{~mm}$ & $U^{*}$ & $4 \times 10^{-11}$ \\
\hline$E^{\prime}$ & $1.17 \times 10^{11} \mathrm{~Pa}$ & $\alpha$ & $2.25 \times 10^{-8} \mathrm{~Pa}^{-1}$ \\
\hline$W^{*}$ & $2.08 \times 10^{-6}$ & $\eta_{o}$ & $1.22 \mathrm{~Pa} \cdot \mathrm{s}$ \\
\hline$a$ & $0.184 \mathrm{~mm}$ & $\check{t}$ & 0.03 \\
\hline$p_{h}$ & $0.54 \mathrm{GPa}$ & $W_{x} / a$ or $W_{y} / a$ & 0.32 \\
\hline
\end{tabular}

Table 2. Input Parameters for the Cases from [37]

\begin{tabular}{|c|c|c|c|}
\hline$R_{x}$ & $12.7 \mathrm{~mm}$ & $u$ & $0.024 \mathrm{~m} / \mathrm{s}$ \\
\hline$E^{\prime}$ & $1.17 \times 10^{11} \mathrm{~Pa}$ & $\alpha$ & $2.37 \times 10^{-8} \mathrm{~Pa}^{-1}$ \\
\hline$w$ & $39.5 \mathrm{~N}$ & $\eta_{o}$ & $1.22 \mathrm{~Pa} \cdot \mathrm{s}$ \\
\hline$a$ & $0.185 \mathrm{~mm}$ & $p_{h}$ & $0.54 \mathrm{GPa}$ \\
\hline
\end{tabular}

Transversal

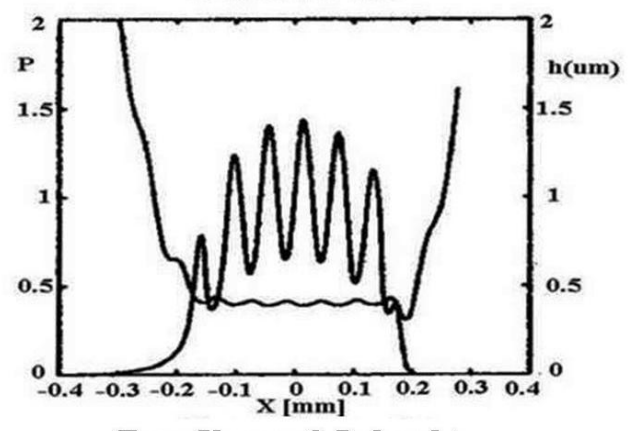

From Venmer \& Lubrecht

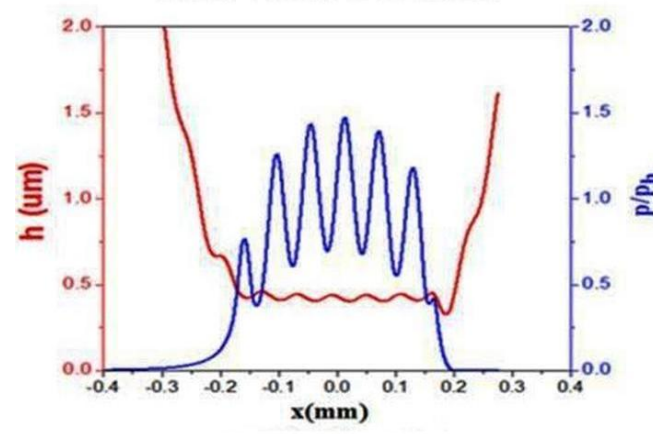

Present solution
Longitudinal

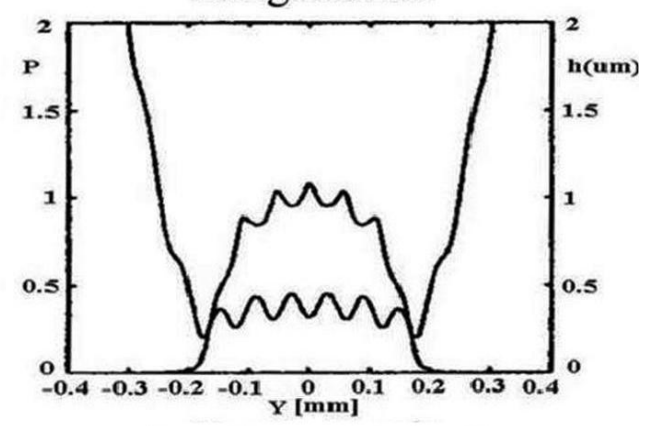

From Venner \& Lubrecht

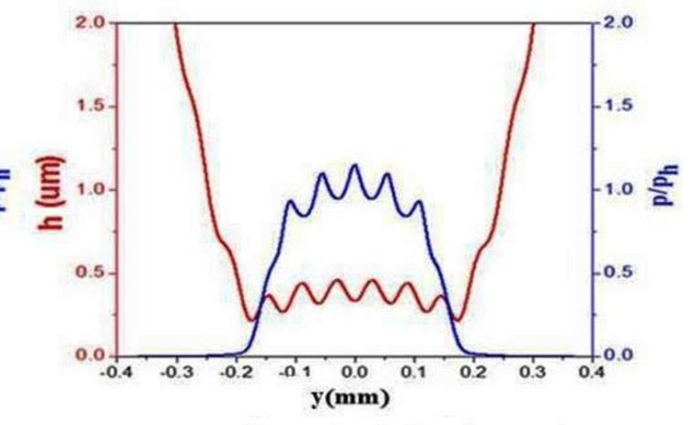

Present solution

Fig. 4. Comparison between Present Solutions and Those from [36].

Left: with transversal waves; right: with longitudinal waves 


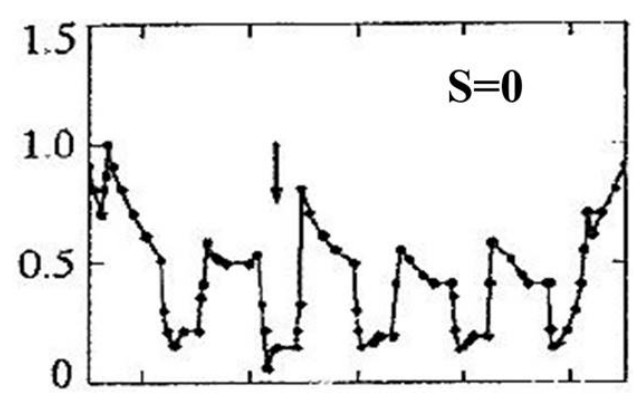

Experimental results

from Kaneta et al.

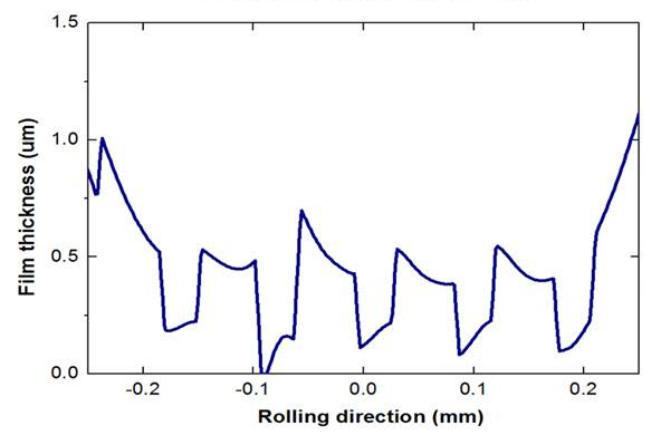

Present solution

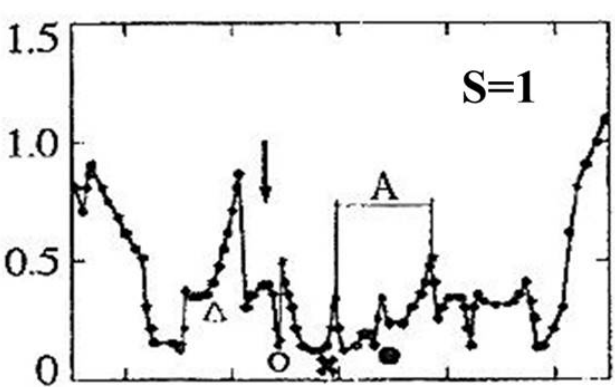

Experimental results from Kaneta et al.

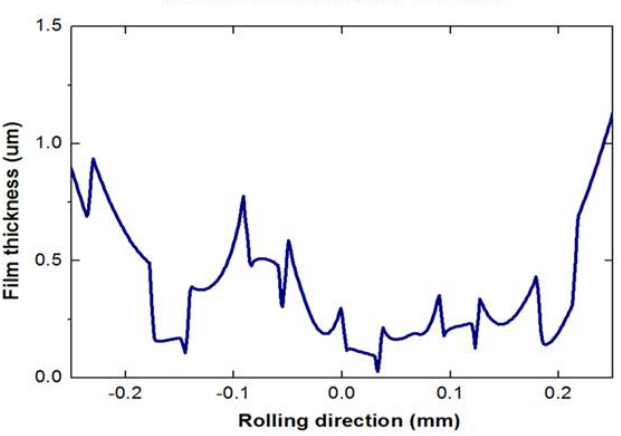

Present solution

Fig. 5. Comparison between Present Solutions and Experimental Data from [37].

Left: pure rolling; right: slide-to-roll ratio $S=1$

Point-contact mixed EHL problems for a ball against a flat are analyzed to demonstrate the application of the model system and the cycle-counting method mentioned in the previous two sections. The ball radius is $R_{x}=R_{y}=19.05 \mathrm{~mm}$. The material properties include effective elastic modulus $E^{\prime}=219.8 \mathrm{GPa}$ and hardness $=7 \mathrm{GPa}$. A typical mineral oil is used, and its properties are: viscosity $\eta_{0}=0.056$ Pa.s, and pressure-viscosity coefficient $\alpha=18.20 \mathrm{GPa}^{-1}$. A normal load of $800 \mathrm{~N}$ is applied, yielding a Hertzian contact radius of $a=0.470 \mathrm{~mm}$ and a maximum Hertzian pressure of $p_{h}=1.727 \mathrm{GPa}$. In all of the cases the rolling speed is set to be $U=0.625 \mathrm{~m} / \mathrm{s}$. The constants in the fatigue life equation (16) are: Weibull slope $e=2.0$, and stress exponent $c=5.0$.

3D rough surfaces with different transversal sinusoidal waves were numerically generated for the study on the influence of sliding on lubrication and fatigue life. Three types of rough surface contacts were simulated: (1) a smooth ball against a flat surface with transversal sinusoidal roughness, (2) two surfaces, both with transversal sinusoidal roughness of the same waviness, and (3) two surfaces, both with transversal sinusoidal roughness of different waviness. All of the surface pairs and their characteristics are given in Table 3. Each pair of the surfaces 
was numerically "tested" under the condition that the flat is moving faster. Note that all the surface pairs have the same composite RMS roughness, 0.50 micron, and each case with sinusoidal waves starts from the in-phase position, i.e. both the wavy surfaces are in the same phase at the starting point.

Table 3. Analyzed Cases with Sinusoidal Surfaces

\begin{tabular}{|c|c|c|c|c|c|c|}
\hline \multirow{2}{*}{ Cases } & \multirow{2}{*}{$\begin{array}{c}\text { Type of } \\
\text { Surfaces }\end{array}$} & \multicolumn{3}{|c|}{ Wavelength } & \multicolumn{2}{|c|}{ Roughness $R_{q}(\mu \mathrm{m})$} \\
\hline & & & $W_{x} / a$ & $W_{y} / a$ & Individual & Composite \\
\hline \multirow{2}{*}{ Case 1} & \multirow{2}{*}{$\begin{array}{l}\text { Ball-smooth, } \\
\text { flat-sinusoidal }\end{array}$} & Ball & $\infty$ & $\infty$ & 0 & \multirow{2}{*}{0.50} \\
\hline & & Flat & 0.25 & $\infty$ & 0.50 & \\
\hline \multirow[b]{2}{*}{ Case 2} & \multirow{2}{*}{$\begin{array}{c}\text { Both sinusoidal } \\
\text { with the same } \\
\text { wavelength }\end{array}$} & Ball & 0.25 & $\infty$ & 0.3536 & \multirow[b]{2}{*}{0.50} \\
\hline & & Flat & 0.25 & $\infty$ & 0.3536 & \\
\hline \multirow[b]{2}{*}{ Case 3} & \multirow{2}{*}{$\begin{array}{l}\text { Both sinusoidal } \\
\text { with different } \\
\text { wavelengths }\end{array}$} & Ball & 0.25 & $\infty$ & 0.3536 & \multirow[b]{2}{*}{0.50} \\
\hline & & Flat & 0.5 & $\infty$ & 0.3536 & \\
\hline
\end{tabular}

\subsection{Peak pressure variation due to sliding}

Sliding significantly affects the lubrication behaviors of the rough surfaces. Figures 6-8 present the variations of the maximum relative pressure influenced by the slide-to-roll ratio, $S$, for Cases 1,2 and 3, respectively. The maximum relative pressure, $P_{\max }$, is dimensionless, defined as the difference between the maximum EHL pressure and the nominal maximum Hertzian pressure divided by the latter. In order to save computing time, the progressive mesh densification (PMD) method, described in detail in [26, 39], is employed for obtaining numerical solutions of mixed EHL with three levels of discretization meshes: Level II corresponding to the mesh of 64x64, Level III for 128x128, and Level IV 256x256. At the beginning of the PMD procedure, level II is employed in order to quickly cancel low frequency errors and get good approximations of the pressure and film thickness distributions. Due to the low mesh density, the computation is very fast. As soon as a relatively loose convergence criterion is met, such as $\varepsilon_{p}=\Sigma\left|P_{i, j}{ }^{\text {new }}-P_{i, j}{ }^{\text {old }}\right| / \Sigma P_{i, j}{ }^{\text {new }}<10^{-4}$, the computation is automatically switched to Level III to focus on the reduction of errors of higher frequencies for a more accurate solution. This procedure is repeated until the proposed ultimate mesh density (Level IV) is reached. On the final mesh level of the highest density, the convergence criteria is tightened, such as $\varepsilon_{p}<10^{-6}$, to ensure high solution accuracy. The solution domain is defined as $-2.5 \leqslant x / a \leqslant 1.5$ and $-2.0 \leqslant$ 
$y / a \leqslant 2.0$, and the dimensionless time step length is set to be $\Delta=U \Delta t / a=0.005$, considerably smaller than the dimensionless mesh spacing of $\Delta x / a=\Delta y / a=0.0156$. The obtained results shown in Figs. 6-8 indicate that a globally stabilized solution can be achieved efficiently. Besides, the higher the $S$ value is, the smaller the number of time steps in the final mesh level (Level IV) for a globally stabilized solution. For Case 1 (smooth ball vs. rough flat), as displayed in Fig. 6, the stabilized value of $P_{\max }$ is the lowest under the pure-rolling condition $(S=0)$, in comparison with those for $S>0$. No matter what $S$ is, the $P_{\max }$ values become nearly constant after stabilization on the highest mesh level, because for every moment of sliding, the characteristics of the interaction between the two surfaces are nearly the same due to the smooth ball surface. Besides, the results show that, when $S$ starts to increase from zero, the $P_{\max }$ value rises up quickly. However, after $S$ goes beyond a certain value, around $S=0.4$ or so in this case, the increase in $P_{\max }$ becomes slower if $S$ is further increased.

On the other hand, for Case 2 (two transversal wavy surfaces with the same wavelength), $P_{\max }$ in the pure rolling contact is nearly constant when the globally stabilized solution is obtained, as demonstrated in Fig. 7. This is because the sinusoidal asperities are always in phase with no relative motion. When sliding is introduced, $P_{\max }$ becomes a function of time, going up and down, because the phase difference between the two transversal wavy surfaces constantly changes. The $P_{\max }$ variation is periodic due to relative sliding, and the period reduces as $S$ increases. However, the average $P_{\max }$ does not seem to increase continuously with $S$; it rises if the $S$ increase is smaller, and then gets stabilized if $S$ increases further in a practically common range to 1.6 analyzed in the current study.

For Case 3 (two transversal waves with different wavelengths), as shown in Fig. 8, the $P_{\max }$ curves exhibit a behavior similar to that in Case 2, except that during the $P_{\max }$ variation, both the maximum of $P_{\max }$ and the amplitudes of $P_{\max }$ oscillations are lower than those in Case 2. This indicates that, with the same RMS roughness, the surface wavelength arrangement has a significant influence on the lubrication characteristics. One can improve the lubrication performance and stability through changing the wavelengths of the rough surfaces. The current mixed EHL model system can be employed for the optimization of surface design in practice. 


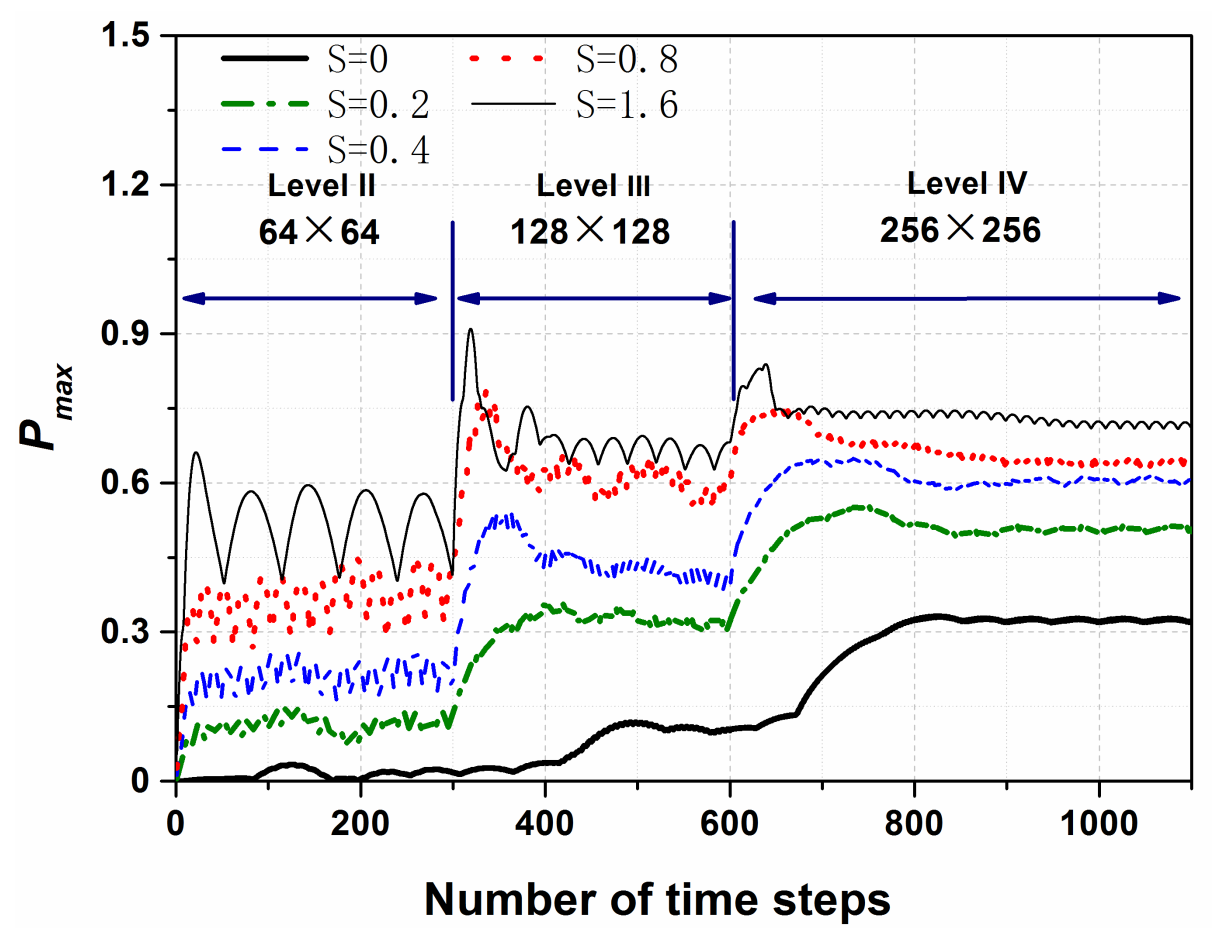

Fig. 6. Variations of $\boldsymbol{P}_{\max }$ at Different $S$ for Case 1.

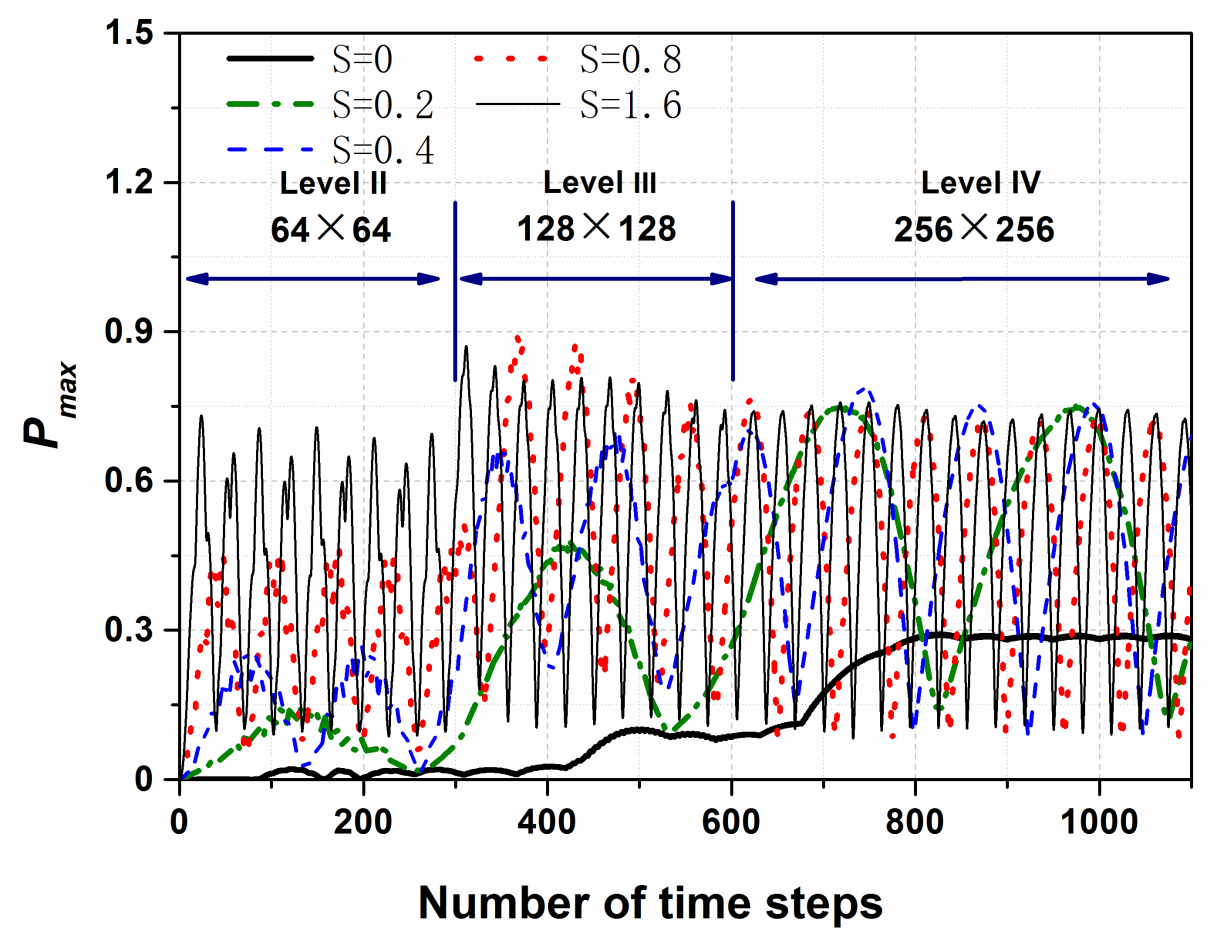

Fig. 7. Variations of $\boldsymbol{P}_{\max }$ at Different $S$ for Case 2. 


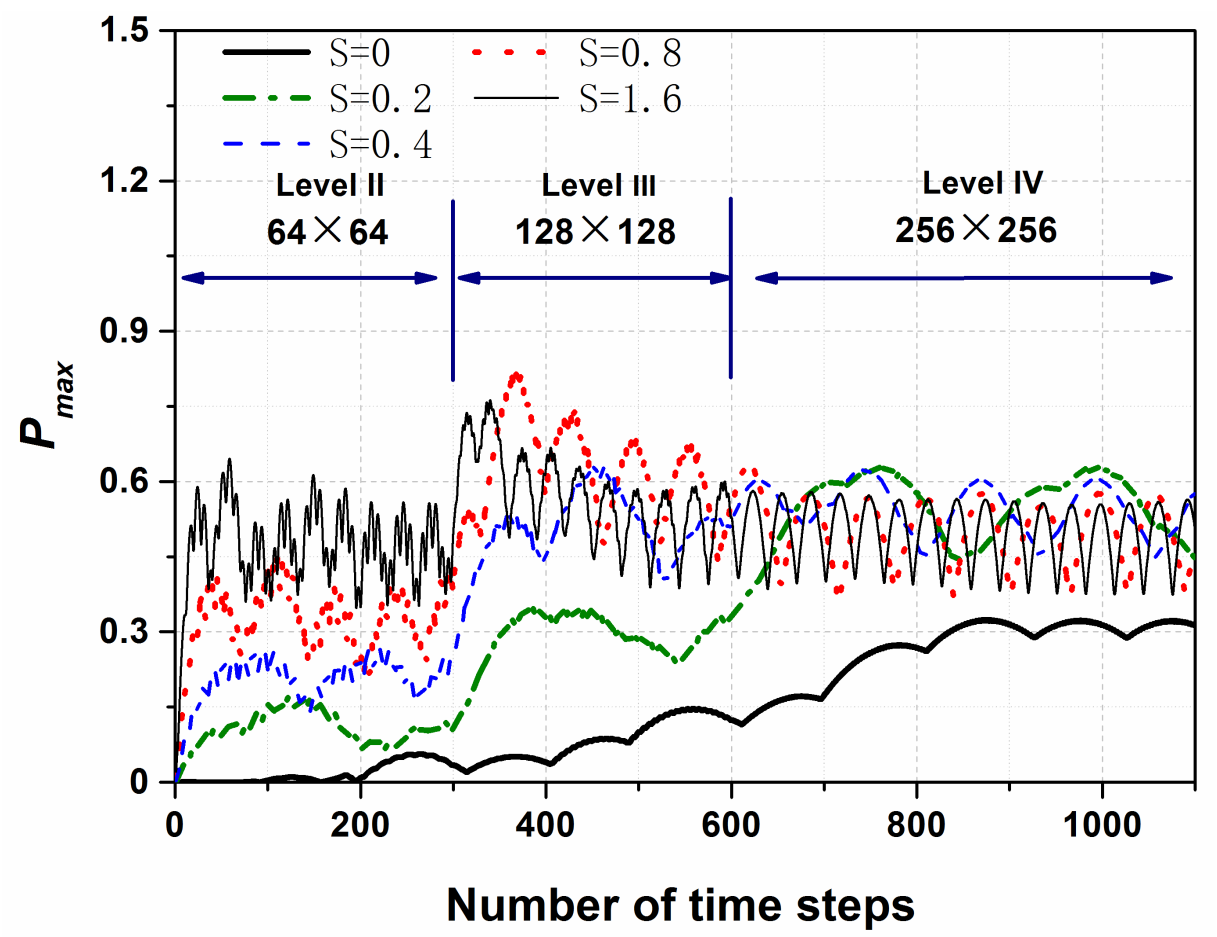

Fig. 8. Variations of $\boldsymbol{P}_{\max }$ at Different $S$ for Case 3.

The dimensionless pressure distributions along the $\mathrm{x}$ direction at different $S$ for Cases 1, 2 and 3 are plotted in Figs. 9, 10 and 11, respectively. These distributions clearly demonstrate the influence of sliding on the asperity peak pressure. Obviously, the pressure peaks due to surface asperities are significantly higher than the maximum Hertzian pressure, no matter how high the sliding is. Also, generally, at high sliding speeds the pressure peaks appear to be considerably higher than that under the pure rolling condition. This increase in the maximum pressure peaks should lead to high subsurface stresses, and thus a significant reduction of the fatigue life. In Case 1, shown in Fig. 9, the pressure distribution at each $S$ is plotted at a certain time step after solution stabilization. The pressure distributions during the interaction between the smooth ball and the rough flat keep nearly the same at each time step, showing nearly constant $P_{\max }$, as illustrated in Fig. 6. In Case 2 and Case 3, however, the $P_{\max }$ variations are periodic due to the relative sliding motion between the two sinusoidal wavy surfaces. The pressure distributions are indeed time-dependent; it varies periodically as well. Note that, since $P_{\max }$ keeps fluctuating and the pressure profile is transient, the curves given in Figs. 10 and 11 are instantaneous samples for reference only, which may or may not represent the specific moments at which the maximum value of $P_{\max }$ occurs. Besides, Figs. 9-11 show that the pressure distributions in Cases 1 and 2 are 
similar to each other in shape with sinusoid-like peaks. However, when the wavelengths of the two rough surfaces are different, the pressure profiles appear to be considerably altered, as shown in Fig. 11, and the peak shape becomes more complex and varies instantaneously.

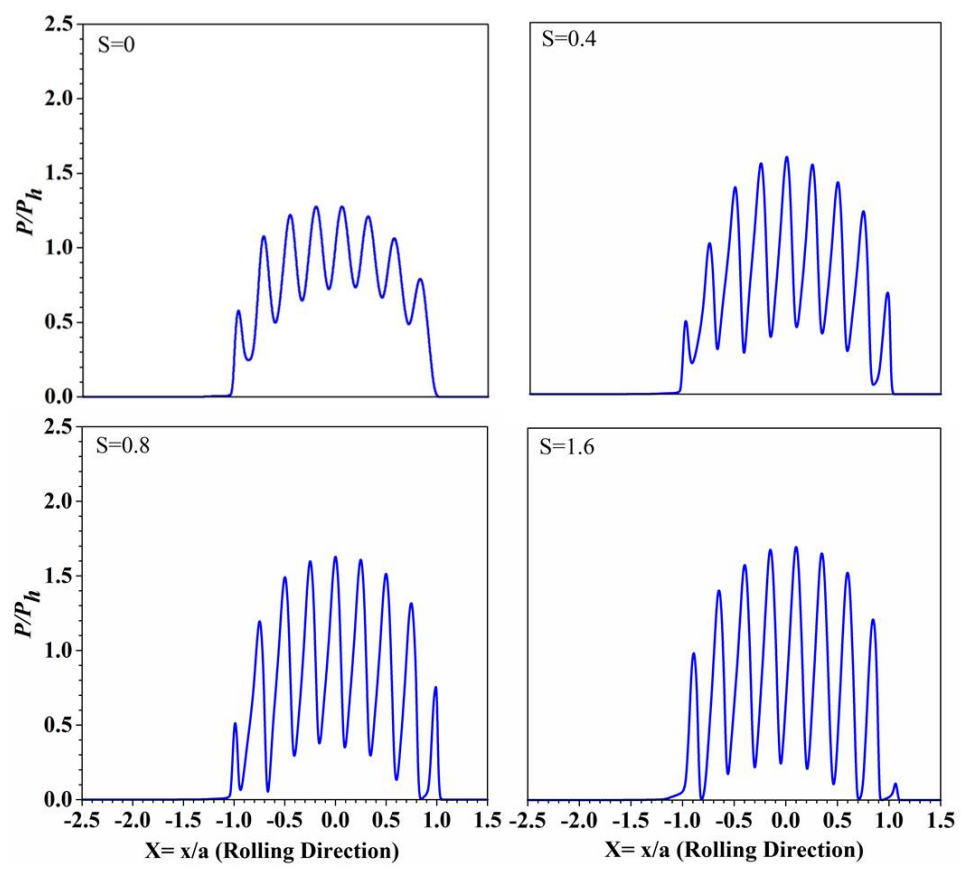

Fig. 9. Pressure Distributions Along the $x$-Axis for Case 1.
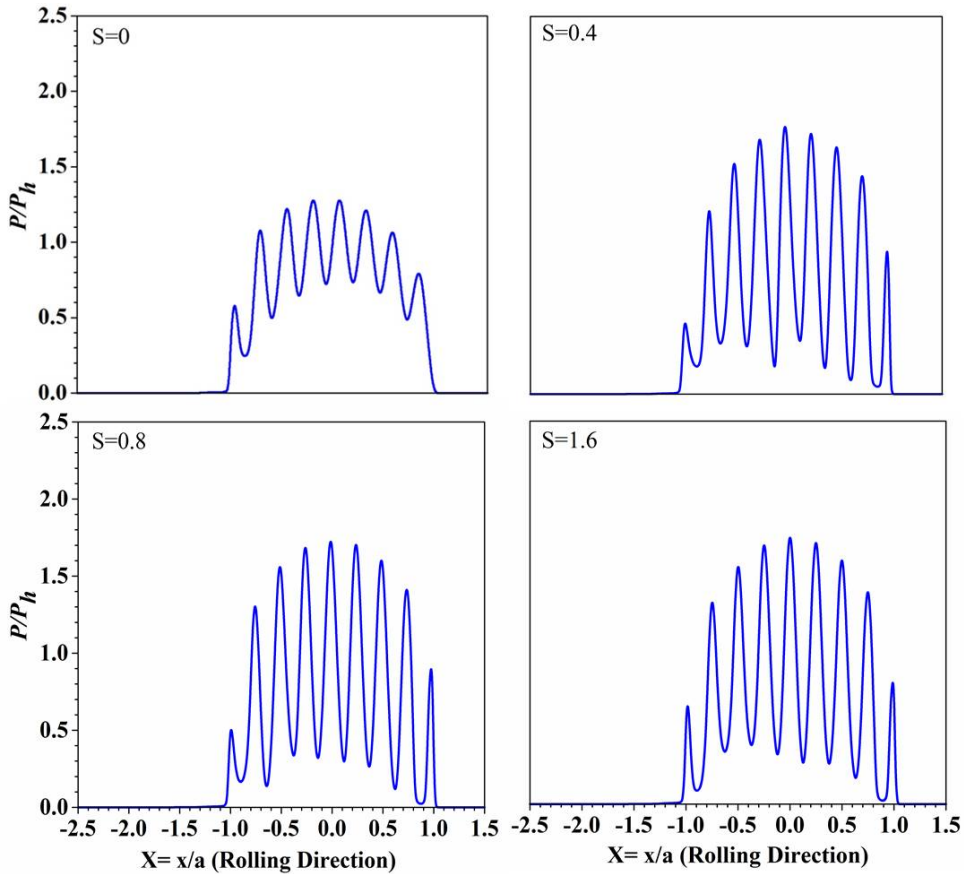

Fig. 10. Pressure Distributions Along the $x$-Axis for Case 2. 


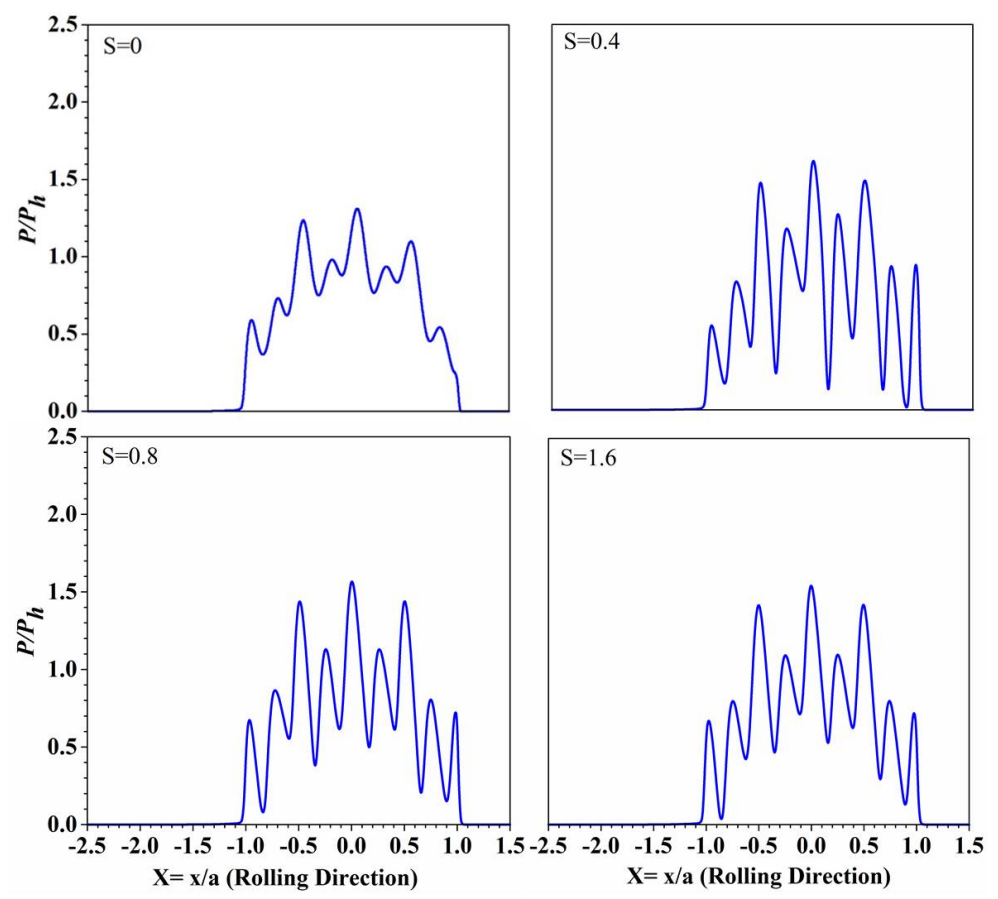

Fig. 11. Pressure Distributions Along the $x$-Axis for Case 3.

\subsection{Subsurface stress variation due to sliding}

The asperities under relative sliding experience the "loading and unloading” process as manifested by the pressure variations. Figure 12, showing the contours of the octahedral stresses along the centerline cross section, presents a set of sample results of the subsurface stress distributions for Case 1 at $S=0,0.4,0.8$ and 1.6, respectively. These contour plots clearly demonstrate the influence of asperity peak pressure on the stresses, indicating that localized high stress concentrations near the surface are induced by the pressure peaks resulted from the moving asperities. When the surfaces are in the pure rolling motion, $S=0$, the near-surface stress appears to be the lowest in comparison with those of $S>0$. When the relative sliding starts to increase from $S=0$, the maximum subsurface stress quickly rises, but its peak value becomes stabilized if $S$ is further increased. This appears to be well correlated with the trend of the maximum pressure peak variation, as demonstrated in Figs. 6 and 9. Note that each contour map in Fig. 12 presents an instantaneous stress distribution at a certain moment, not the stress cycle counting results during a period of time. For Cases 2 and 3, the subsurface stress fields at different $S$ values are also correlated directly with the pressure distributions in a similar way although the results are not plotted here. Note that because the calculated friction coefficients are small, the stress contours are still largely symmetric, and the subsurface stress contours for the cases of $S=1.6$ 
with and without considering the effect of friction are similar, as shown by the two bottom-right plots in Fig. 12.
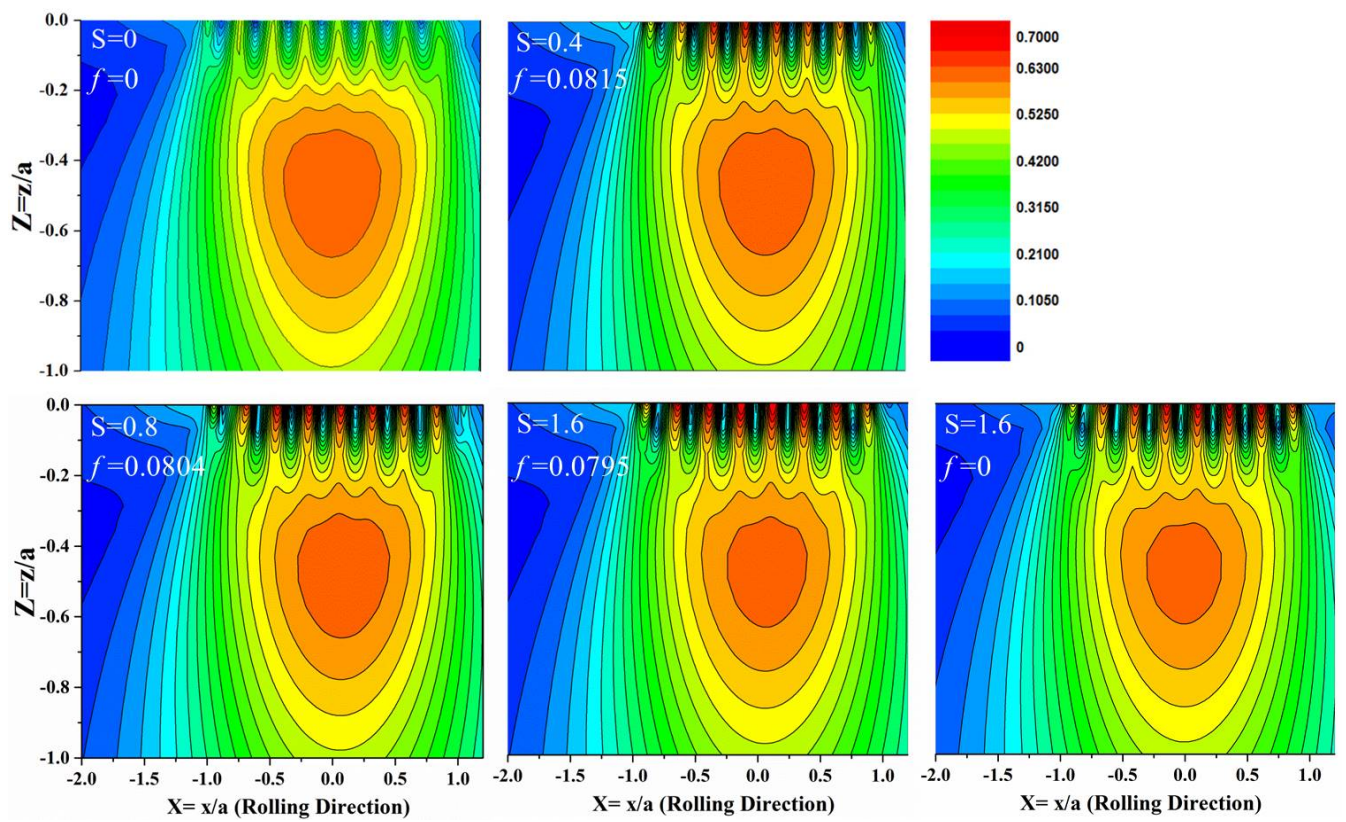

Fig. 12. Octahedral Stresses Distribution on the $x-z$ Cross Section for Case 1.

\subsection{Influence of sliding on fatigue life}

The fatigue life predictions for the ball surface at different $S$ are illustrated in Figs. 13-15, in which all the life values are relative in comparison with a reference that is obtained from Case 1 under the pure-rolling condition. Here, the life data with and without the cycle counting adjustment are both presented for 50\% survival probability. For clarity, the results of the maximum value of $P_{\max }$ are also included in Figs. 13-15. Note that in these figures, the sliding friction coefficient is treated as zero under the pure-rolling contact condition. The friction coefficient increases rapidly when $S$ is increased from 0 ; when $S$ is beyond a certain value, for example, $S=0.2$, the friction coefficient decreases as $S$ is further increased.

It is important to note that significant fatigue life reductions, illustrated in Figs. 13-15, in comparison with the reference, are caused by the two physical mechanisms as anticipated before: the increase in stress cycle number due to the surface asperities in relative sliding motion, and the increase in the maximum pressure peak height (and, consequently, the maximum subsurface stress) resulted from the moving asperities in the mixed EHL system. 
Note that without considering the stress cycle counting, Eq. (4), the predicted fatigue life time depends exclusively on the fatigue integral in the right-hand side of Eq. (16). This integral is calculated by integrating the stresses inside the stressed volume, $V$. The high pressure peaks on the surface due to the moving rough surface asperities usually produce correspondingly high interior stress peaks, which certainly have a strong influence on the resultant fatigue integral, even though all of the stresses in $V$ are used to calculate the integral. When the stress cycle counting adjustment is employed, resultant cycle number $M$ from Eq. (16) is considered as $M=$ $N \times n$, in which $N$ is the number of component loading cycles and $n$ the number of asperity contacts per loading cycle experienced by the surface and evaluated by Eq. (4). Obviously a large $n$ would certainly lead to a reduced loading cycle number $N$ thus a significant life time reduction.

The prediction results for Case 1 summarized in Fig. 13 show that when the stress cycle counting is not considered, the relative fatigue life gradually decreases if $S$ increases. The life becomes stable if $S$ is beyond 0.40 or so, and this well correlates with the trend of $P_{\max }$ variation. Note that even at $S=1.6$, the life reduction is only around $12.9 \%$, in comparison with the reference life at $S=0$. This behavior is directly due to the second mechanism stated above. When the stress cycle counting is also considered, more significant life time reduction can be seen at each none zero $S$ value. In fact, the contribution to the life reduction due to the first mechanism (cycle increase) appears to be greater than that due to the second mechanism (pressure peak increase), especially at high slide-to-roll ratios. The total life reduction for the case of $S=1.6$ can be as large as $98 \%$ for Case 1.

The predicted life data for Case 2 are plotted in Fig. 14. It is evident that when the cycle counting adjustment is not used, the predicted life decreases quickly in the beginning and then becomes stabilized beyond $S=0.20-0.25$ or so if $S$ continuously increases. This well correlates with the trend of the maximum pressure peak height variation shown in Figs. 7 and 10. If the stress cycle counting is included in the predictions, the fatigue life values are further reduced significantly, as demonstrated in Fig. 14. For the case of $S=0.8$, the second mechanism may cause a life decrease as great as about $21 \%$ in comparison with the reference, while the first mechanism can result in an additional reduction up to about $72.2 \%$ as compared with that without considering the cycle counting. The maximum total life reduction at $S=1.6$ can reach 98.8\% for Case 2, in which both the transversal sinusoidal surfaces have the same wavelength. 


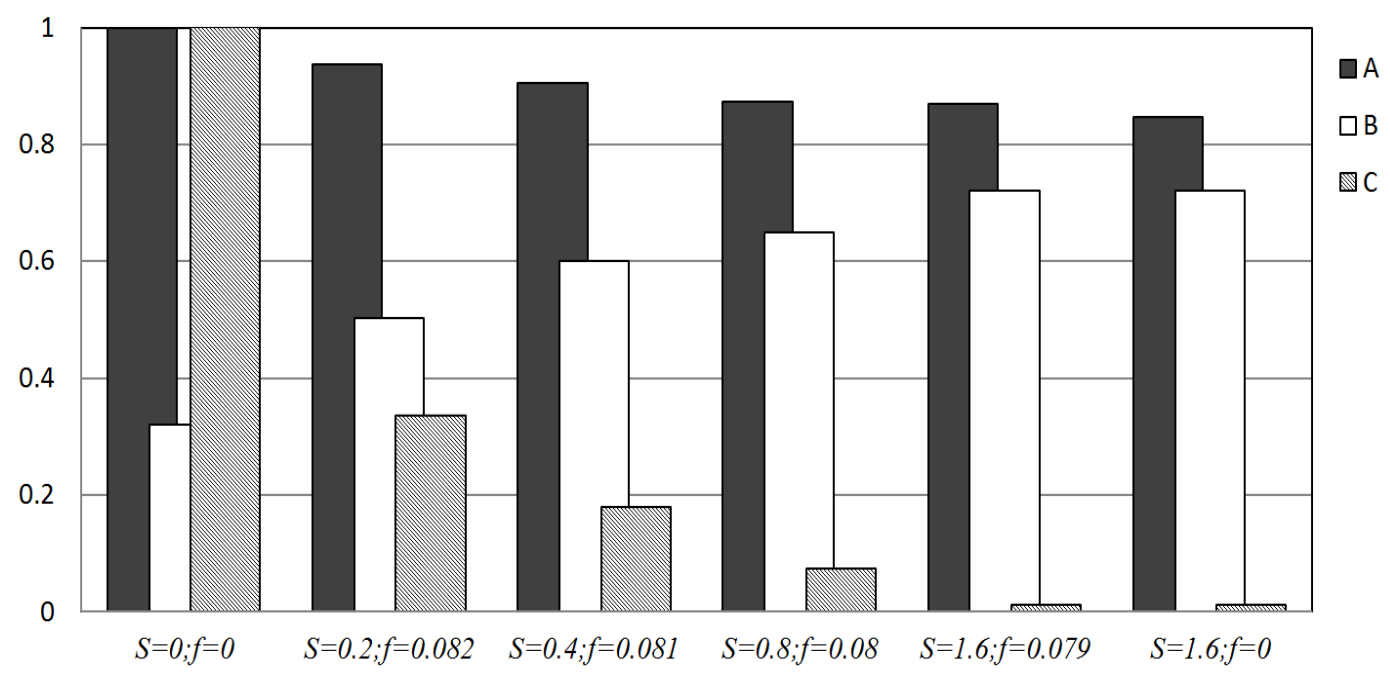

Fig.13 Fatigue Lives of the Ball Surface at Different $S$ and for Case 1. Friction Coefficient $f$ for $\mathbf{S}>\mathbf{0}$ is Non-Zero.

(A: life without stress cycle counting; $\mathrm{B}: P_{\max }$; : life with stress cycle counting)

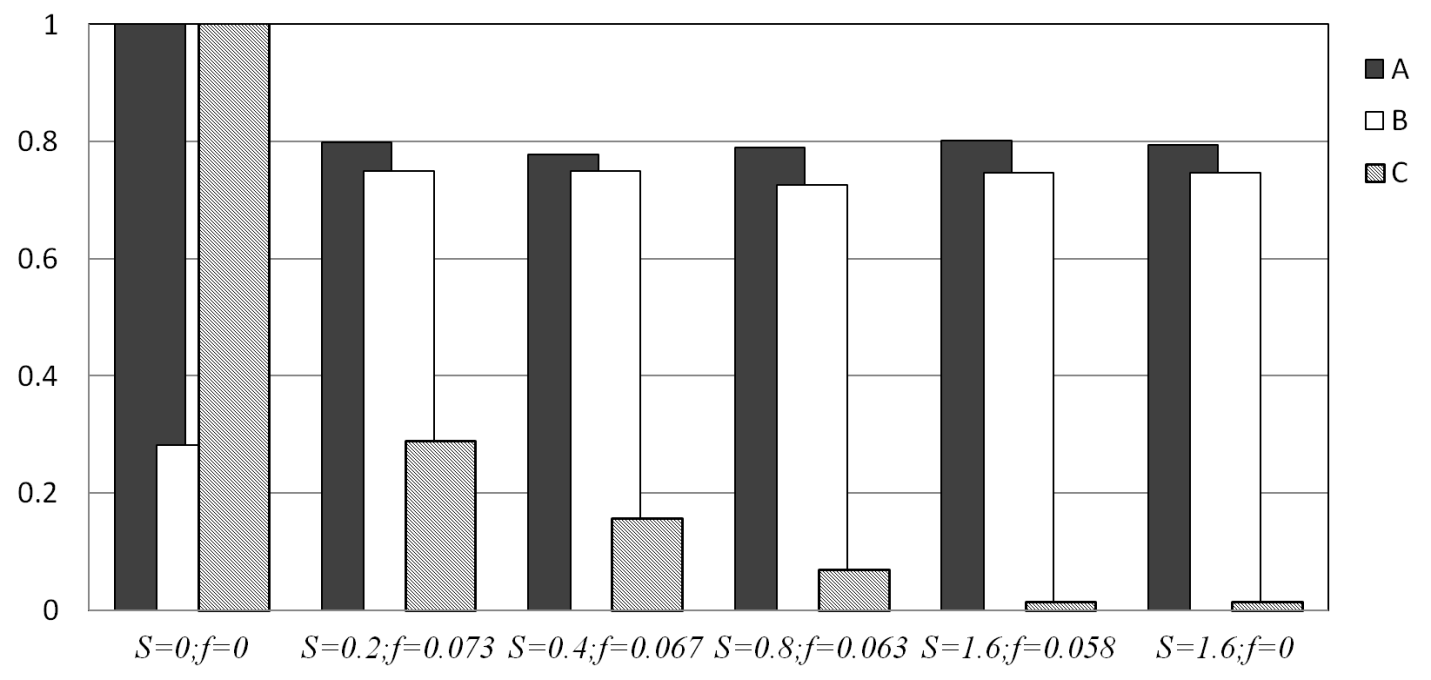

Fig. 14. Fatigue Life of the Ball Surface at Different $S$ for Case 2. Friction Coefficient $f$ for $\mathrm{S}>\mathbf{0}$ is Non-Zero.

(A: life without stress cycle counting; B: $P_{\max }$; : life with stress cycle counting) 


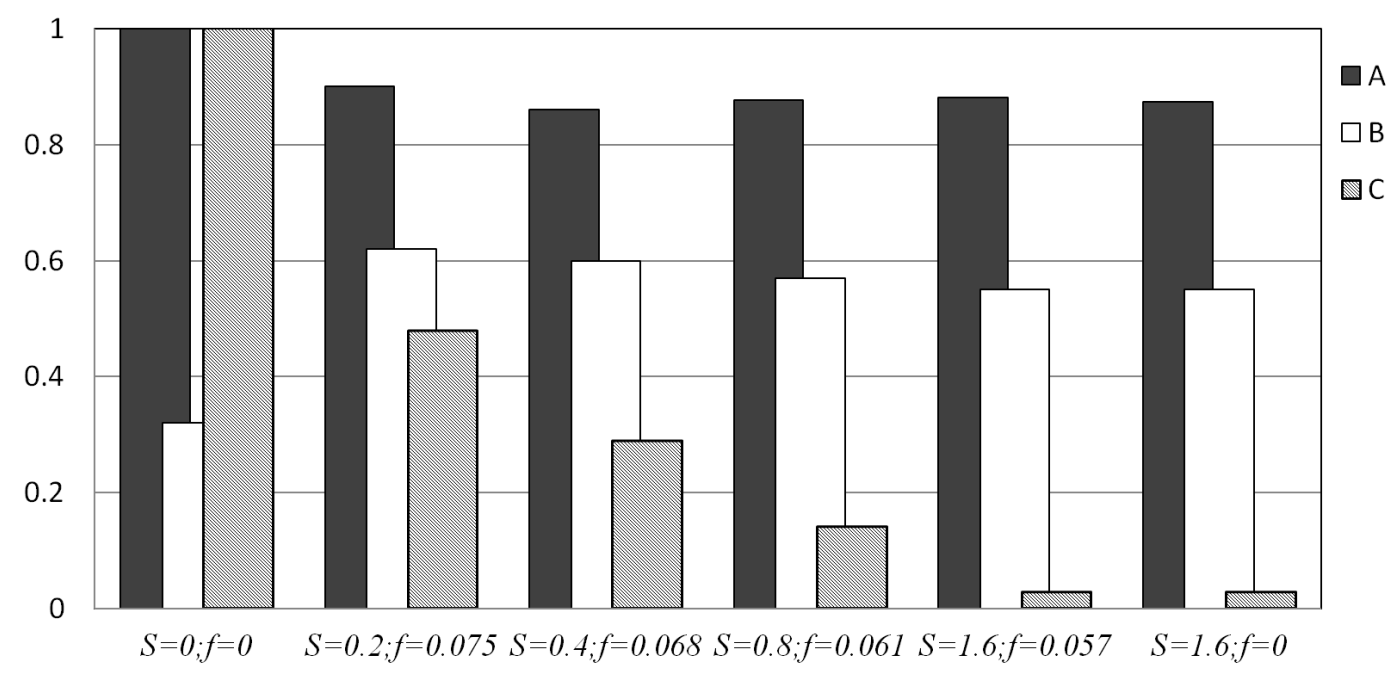

Fig. 15. Fatigue Life of the Ball Surface at Different $S$ for Case 3. Friction Coefficient $f$ for $\mathrm{S}>\mathbf{0}$ is Non-Zero.

(A: life without stress cycle counting; B: $P_{\max } ; \mathrm{C}$ : life with stress cycle counting)

For Case 3, in which the two surfaces have different wavelengths, the basic trend for the life reduction due to the sliding motion is similar to that for Case 2. However, the maximum reduction appears to be only about $11 \%$ when the cycle counting is not considered, and the maximum total reduction is about $97.3 \%$ at $S=1.6$ with the usage of the cycle counting method. Obviously, it is desirable to make the two surfaces have different wavelengths. If the fatigue life of Surface 1 is concerned in practice, it is preferred to have a longer wavelength for Surface 2. In this way the maximum pressure peak height and the maximum subsurface stress can be lower than those for the two surfaces having the same wavelength. In addition, the stress cycle number should be considerably smaller as well. Therefore, one may improve the component service life through optimizing the wavelengths of the rough surfaces while keeping the same roughness, especially when a high relative sliding motion is involved in the mixed EHL conjunction.

The experiments found in literature qualitatively support the proposed mechanism of contact fatigue failure acceleration by sliding. The experimental results from the two-disc experiments obtained by Rabaso et al. [40] showed that the increase in sliding ratio from 0.06 to 0.2 and then to 0.4 resulted in 2.8 and 3.4 times total pitting area increase, respectively. The present study suggests that the same amount of changes in sliding ratio can lead to the reduction of fatigue life in about 2.4 and 4.5 time, respectively. Similarly, Nakajima's experiments [41] 
showed the increase in sliding ratio from $0.5 \%$ to $0.7 \%$ and $3.2 \%$ can lead to the life decrease as great as about $36.8 \%$ and $82.7 \%$, respectively, under a heavier load of 3.4 4.2 GPa Hertz pressure, as compared to the fatigue life reduction of about $1.14 \%$ and $14.5 \%$, respectively, in the current calculations. Note that Nakajima's experiments involved much harsher conditions in terms of load and lubrication.

The effect of friction, for these lubricated contacts, on the contact fatigue life reduction are also studied by comparing the results for $S=1.6$ with and without considering the friction coefficient, as shown in Figs. 13-15. The results suggest that it is proper and computationally effective to ignore the effect of friction in the analysis of contact fatigue of lubricated surfaces. However, the current model does not consider the wear effect of frictional rubbing.

\section{CONCLUSIONS}

The effect of relative sliding motion on the contact fatigue life of surfaces with sinusoidal roughness is modeled and numerically investigated. A set of simple equations for asperity stress cycle counting is derived. Mixed EHL simulations are executed to reveal the variations of the mixed EHL pressure distribution and the subsurface stress as functions of the slide-to-roll ratio, $S$. Fatigue life predictions are conducted for typical sample cases by using the Zaretsky contactfatigue life model and the proposed stress-cycle-counting formulae. The following conclusions can be drawn from the numerical results:

(1) Generally, the maximum peak height of mixed EHL pressure, as well as the maximum subsurface stress, increase considerably with $S$ when it is raised from zero. However, the increase in these peak values levels as sliding is further increased.

(2) The number of stress cycles experienced by a certain piece of the surface material also increases with relative sliding. It is generally a function of $S$ and surface asperity density of the mating surface.

(3) For cases in which both the surfaces are rough, the $P_{\max }$ variation is periodic due to relative sliding. The periodicity reduces as $S$ increases.

(4) The friction coefficient increases rapidly with the increase in slide to roll ratio when the latter is small. It decreases if the slide-to-roll ratio is further increased. The friction 
coefficient in mixed lubrication is generally small, and its influences on the subsurface stress and contact fatigue life are also limited. It is proper and computationally effective to ignore the effect of friction in the analysis of rolling-sliding contact fatigue of lubricated surfaces.

(5) Relative sliding between two rough surfaces may cause significant contact fatigue life reduction due to both the increased number of asperity stress cycles and the promoted pressure peaks.

(6) One may be able to improve the service life of components under a rolling-sliding condition through optimizing the wavelengths of the two rough surfaces without reducing the composite RMS roughness. Generally, if the fatigue life of one surface is concerned, a topography of a longer wavelength of its mating surface asperities is preferred. The present model can be employed as a useful tool for the surface design optimization in practice.

\section{Acknowledgements}

The present study is partially supported by NSFC (National Science Foundation of China) Projects \#51435001 and \#51375506. The support from the State Key Laboratory of Mechanical Transmission at Chongqing University (\#0301002109159 and SKLMT-ZZKT-2012 ZD 03) is also acknowledged. W. Pu would also like to acknowledge the research foundation from Sichuan University (\#SCUMIAOZI2014-1-1).

\section{Nomenclature}

$a \quad=$ radius of Hertzian contact circle

A, $\mathscr{\mathscr { t }}=$ amplitude and dimensionless amplitude of sinusoidal waves, $\mathscr{\mathscr { t }}=A R_{x} / a^{2}$

$c \quad=$ stress exponent

e $\quad=$ Weibull slope

$E^{\prime} \quad=$ effective elastic modulus

$f_{1}, f_{2}=$ asperity densities of Surfaces 1 and 2, respectively

$G^{*} \quad=\alpha E^{\prime}$, dimensionless material parameter

$h \quad=$ local film thickness 


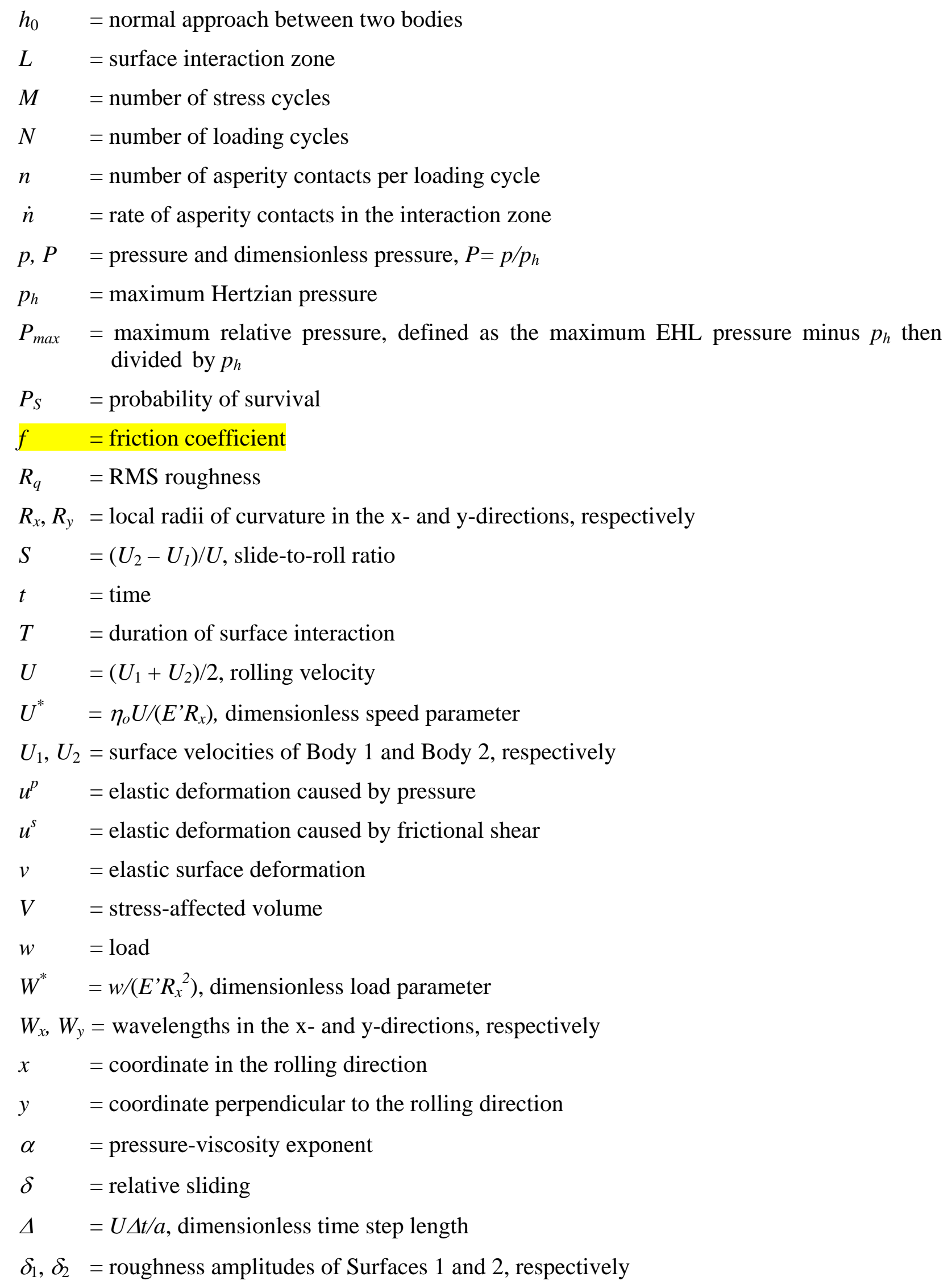




$$
\begin{array}{ll}
\eta & =\text { lubricant viscosity } \\
\eta_{0} & =\text { ambient viscosity at inlet temperature } \\
\eta^{*} & =\text { effective viscosity } \\
\rho & =\text { lubricant density } \\
\rho_{0} & =\text { ambient lubricant density at inlet temperature } \\
\sigma_{\text {eff }} & =\text { effective stress } \\
\tau_{0} & =\text { reference shear stress } \\
\tau_{1} & =\text { shear stress on Surface } 1
\end{array}
$$

\section{References}

[1] Weibull, W., 1939, “A Statistical Theory of the Strength of Materials,” Proceedings 151, Royal Swedish Academy of Engineering Sciences.

[2] Weibull, W., 1939, “The Phenomenon of Rupture in Solids,” Proceedings 153, Royal Swedish Academy of Engineering Sciences.

[3] Bakharev, V. M., 1945, “On the Fatigue of Steel under Repeated Overloading,” Proceed. Central Institute of Aviation Motors (TsIAM),” 91, Oborongiz, Moscow (in Russian).

[4] Lundberg, G., and Palmgren, A., 1947, "Dynamic Capacity of Rolling Bearings,” Acta Polytechnica, Mech. Eng. Series I, Royal Swedish Academy of Engineering Sciences, \#3, 1, pp. 1-50.

[5] Ioannides, E. and Harris, T. A., 1985, “A New Fatigue Life Model for Rolling Bearings”, ASME J. Tribol., 107, pp. 367-378.

[6] Bujold, M. P., Zhu, D., Epstein, D., Wang, Q., and Keer, L. M., 2004, “ Investigation of Sliding/Rolling Contact Fatigue Life with Both Two Disk Experiments and Computer Model Based Prediction,” presented at the STLE 2004 Annual Meeting, May 19, 2004, Toronto, Canada.

[7] Govindarajan, N., and Gnanamoorthy R., 2007, "Rolling/Sliding Contact Fatigue Life Prediction of Sintered and Hardened Steels,” Wear, 262(1), pp.70-78.

[8] Oksanen, V., Valtonen, K., Andersson, P., et al., 2015, “Comparison of laboratory rollingsliding wear tests with in-service wear of nodular cast iron rollers against wire ropes", Wear, 340, pp. 73-81.

[9] Ramalho, A., Esteves, M., and Marta, P., 2013, "Friction and Wear Behavior of RollingSliding Steel Contacts”, Wear, 302(1), pp.1468-1480.

[10] Lee, D., H., Seo, J., W., and Kwon., S., J., 2014, “Numerical Analysis of the Effect of Slip Ratio on the Fatigue Crack Initiation Life in Rolling Contact," Advanced Materials Research. 891, pp. 1791-6. 
[11] Seo, J. W., Jun, H. K., Kwon, S. J., et al., 2016, "Rolling contact fatigue and wear of two different rail steels under rolling-sliding contact”, International Journal of Fatigue, 83, pp.184-194.

[12] Zaretsky, E. V., 1987, “Fatigue Criterion to System Design, Life and Reliability,” J. Propulsion and Power, 3(1), pp. 76-83.

[13] Blake, J. and Cheng, H. S., 1991, “A Surface Pitting Life Model for Spur Gears, 1. Life Prediction,” ASME J. Tribol., 113, pp. 712-718.

[14] Kudish, I. I., and Burris, K. W., 2000, "Modern State of Experimentation and Modeling in Contact Fatigue Phenomenon: Part II - Analysis of the Existing Statistical Mathematical Models of Bearing and Gear Fatigue Life. New Statistical Model of Contact Fatigue”, Tribol. Trans., 43, pp. 293-301.

[15] Kim, T. H. and Olver, A. V., 1998, "Stress History in Rolling-Sliding Contact of Rough Surfaces,” Tribol. Int., 31, pp. 727-736.

[16] Hanson, M. T., and Keer, L. M., 1992, “An Analytical Life Prediction Model for Fatigue Crack Propagation in Contact Fatigue,” Tribol. Trans., 35, pp. 451-461.

[17] Ai, X. L., 1998, "Effect of Three-Dimensional Random Surface Roughness on Fatigue Contact”, ASME J. Tribol., 120, pp. 159-164.

[18] Xu, G., and Sadeghi, F., 1996, “Thermal EHL Analysis of Circular Contacts With Measured Surface Roughness,” ASME J. Tribol., 118, pp. 473-483.

[19] Zhu, D., and Ai, X. L., 1997, "Point Contact EHL Based on Optically Measured ThreeDimensional Rough Surfaces,” ASME J. Tribol., 119, pp. 375-384.

[20] Jiang, X., Hua, D. Y., Cheng, H. S., Ai, X. L., and Lee, S. C., 1999, “A Mixed Elastohydrodynamic Lubrication Model With Asperity Contact,” ASME J. Tribol., 121, pp. 481-491.

[21] Holmes, M. J. A., Qiao, H., Evans, H. P., and Snidle, R. W., 2004, "Surface Contact and Damage in Micro-EHL," Life Cycle Tribology, Proceedings of the 31st Leeds-Lyon Symposium on Tribology, Leeds 2004, Tribology and Interface Engineering Series, Elsevier, Amsterdam, pp. 605-616.

[22] Holmes, M. J. A., Evans, H. P., and Snidle, R. W., 2005, “Analysis of Mixed Lubrication Effects in Simulated Gear Tooth Contacts,” ASME J. Tribol., 127, pp. 61-69.

[23] Zhu, D., Liu, Y., and Wang, Q., 2014, “On the Numerical Accuracy of Rough Surface EHL Solution,” Tribol. Trans., 57, pp. 570-580.

[24] Zhu, D., and Hu, Y. Z., 1999, "The Study of Transition From Full Film Elastohydrodynamic to Mixed and Boundary Lubrication," The Advanced Frontier of Engineering Tribology, Proceedings of 1999 STLE/ASME H. S. Cheng Tribology Surveillance, STLE/ASME, Park Ridge, IL, pp. 150-156.

[25] Hu, Y. Z., and Zhu, D., 2000, “A Full Numerical Solution to the Mixed Lubrication in Point Contacts,” ASME J. Tribol., 122, pp. 1-9.

[26] Zhu, D., 2007, “On Some Aspects in Numerical Simulation of Thin Film and Mixed EHL,” Proc. IMechE, Part J: J. Eng. Tribol., 221, pp. 561-579. 
[27] Epstein, D., Zhu, D., Keer, L. M., Wang, Q., and Cheng, H. S., 2003, "Effect of Surface Topography on Contact Fatigue in Mixed Lubrication,” Tribol. Trans., 46, pp. 506-513.

[28] Zhu, D., Ren, N., and Wang, Q., 2009, "Pitting Life Prediction Based on a 3-D Line Contact Mixed EHL Analysis and Subsurface von Mises Stress Calculation,” ASME J. Tribol., 131, pp.041501 1-8.

[29] Greco, A., Martini, A., Liu, Y., Lin, C., and Wang, Q., 2010, "Rolling Contact Fatigue Performance of Vibro-Mechanical Textured Surfaces," Tribology Transactions, Vol. 53, pp. 610-620.

[30] Martini, A., Zhu, D., and Wang, Q., 2007, "Friction Reduction in Mixed Lubrication," Tribology Letters, 28(2), pp. 171-181.

[31] Bair, S., and Winer, W. O., 1979, "A Rheological Model for Elastohydrodynamic Contacts Based on Primary Laboratory Data,” ASME J. Tribol., 101(3), pp. 258-264.

[32] Zhu, D., and Cheng, H.S., 1989, "An Analysis and Computational Procedure for EHL Film Thickness, Friction and Flash Temperature in Line and Point Contacts,” Tribol. Trans., 32, pp. 364-370.

[33] Johnson, K. L., 1985, “Contact Mechanics,” Cambridge University Press, London.

[34] Polonsky, I. A. and Keer, L. M., 1999, “A Numerical Method for Solving Rough Contact Problems Based on the Multi-Level Multi-Summation and Conjugate Gradient Techniques,” Wear, 231, pp. 206-219.

[35] Zhu, D., and Hu, Y. Z., 2001, "A Computer Program Package for the Prediction of EHL and Mixed Lubrication Characteristics, Friction, Subsurface Stresses and Flash Temperatures Based on Measured 3-D Surface Roughness," Tribol. Trans., 44, pp. 383-390.

[36] Venner, C. H., and Lubrecht, A. A., 1996, "Numerical Analysis of the Influence of Waviness on the Film Thickness of a Circular EHL Contact," ASME J. Tribol., 118, pp. 153-161.

[37] Kaneta, M., Tani, N., and Nishikawa, H., 2002, "Optical Interferometric of Observations of the Effect of Moving Transverse Asperities on Point Contact EHL Films," Proc. the 29th Leeds-Lyon Symp. on Tribol., Elsevier, Amsterdam, pp. 101-109.

[38] Liu, Y.C., Wang, Q., Zhu, D., Wang, W.Z., and Hu, Y.Z., 2009, "Effect of Differential Scheme and Viscosity Model on Rough Surface Point Contact Isothermal EHL,” ASME J. Tribol., 131,pp. 044501 1-5.

[39] Pu, W., Wang J.X., and Zhu D., 2016, "Progressive Mesh Densification Method for Numerical Solution of Mixed Elastohydrodynamic Lubrication,” ASME J. Tribol., 138, pp. 021502 1-11.

[40] Rabaso P., Gauthier T., Diaby M., et al., 2013, "Rolling Contact Fatigue: Experimental Study of the Influence of Sliding, Load, and Material Properties on the Resistance to Micropitting of Steel Discs,” Tribology Transactions, 56(2), pp. 203-214.

[41] Nakajima A., and Mawatari T., 2006, "Effect of Slip Ratio on Rolling Contact Fatigue of Bearing Steel Rollers Lubricated with Traction Oil," IUTAM Symposium on Elastohydrodynamics and Micro-elastohydrodynamics. Springer Netherlands, pp. 333-344. 


\section{List of Figures}

Fig. 1 Contact of Equivalent Cylindrical (or Spherical, or Elliptical) Rollers

Fig. 2 A Sample Ground Surface

Fig. 3 Effect of Relative Sliding on Asperity Cycle Counting

Fig. 4 Comparison Between Present Solutions and Those from [36]

Left: with transversal waves; right: with longitudinal waves

Fig. 5 Comparison between Present Solutions and Experimental Data from [37]

Left: pure rolling; right: slide-to-roll ratio $S=1$

Fig. 6 Variations of $P_{\max }$ at Different Slide-to-Roll Ratios for Case 1

Fig. 7 Variations of $P_{\max }$ at Different Slide-to-Roll Ratios for Case 2

Fig. 8 Variations of $P_{\max }$ at Different Slide-to-Roll Ratios for Case 3

Fig. 9 Pressure Distributions Along the X-Axis for Case 1

Fig. 10 Pressure Distributions Along the $\mathrm{x}$-Axis for Case 2

Fig. 11 Pressure Distributions Along the $\mathrm{x}$-Axis for Case 3

Fig. 12 Octahedral Stresses Distribution on the x-z Cross Section for Case 1

Fig. 13 Fatigue Lives of the Ball Surface at Different $S$ and for Case 1. Friction Coefficient $f$ for $\mathrm{S}>0$ is Non-Zero.

(A: life without stress cycle counting; B: $P_{\max }$; C: life with stress cycle counting)

Fig. 14 Fatigue Life of the Ball Surface at Different Slide-to-Roll Ratios for Case 2. Friction Coefficient $f$ for $\mathrm{S}>0$ is Non-Zero.

(A: life without stress cycle counting; B: $P_{\max } ; \mathrm{C}$ : life with stress cycle counting)

Fig. 15 Fatigue Life of the Ball Surface at Different Slide-to-Roll Ratios for Case 3. Friction Coefficient $f$ for $\mathrm{S}>0$ is Non-Zero.

(A: life without stress cycle counting; B: $P_{\max } ; \mathrm{C}$ : life with stress cycle counting)

\section{List of Tables}

Table 1 Input Parameters for the Cases from [36]

Table 2 Input Parameters for the Cases from [37]

Table 3 Analyzed Cases with Sinusoidal Surfaces 